\title{
Sleep Apnea and Risk of Panic Disorder
}

\author{
Vincent Yi-Fong Su, MD ${ }^{1-3,9^{*}}$ \\ Yung-Tai Chen, $M D^{3,4^{*}}$ \\ Wei-Chen Lin, $M D^{1,3,5}$ \\ $\mathrm{Li}-\mathrm{An} W u, M D^{6}$

\begin{abstract}
PURPOSE Epidemiological studies have identified a trend in the development of depressive and anxiety disorders following a diagnosis of sleep apnea. The relationship between sleep apnea and subsequent panic disorder, however,
\end{abstract} \\ remains unclear.
}

Sbi-Cbuan Chang, $P b D^{1-3,7}$

Diabn-Warng Perng, $\mathrm{PbD}^{1-3}$

Wei-Juin Su, MSc $c^{2,3}$

Yub-Min Chen, $P b D^{2,3}$

Tzeng-Ji Chen, $\mathrm{PbD}^{3,8}$

$Y u-C b i n$ Lee, $M D^{2,3,9}$

Kun-Ta Chou, MD 1-3,9

${ }^{1}$ Center of Sleep Medicine, Taipei Veterans General Hospital, Taipei, Taiwan

${ }^{2}$ Department of Chest Medicine, Taipei Veterans General Hospital, Taipei, Taiwan

${ }^{3}$ School of Medicine, National Yang-Ming University, Taipei, Taiwan

${ }^{4}$ Department of Medicine, Heping-Fuyou Branch, Taipei City Hospital, Taipei, Taiwan

${ }^{5}$ Department of Psychiatry, Taipei Veterans General Hospital, Taipei, Taiwan

${ }^{6}$ Department of Radiology, Heping-Fuyou Branch, Taipei City Hospital, Taipei, Taiwan

${ }^{7}$ Institute of Emergency and Critical Care Medicine, School of Medicine, National Yang-Ming University, Taipei, Taiwan

${ }^{8}$ Department of Family Medicine, Taipei Veterans General Hospital, Taipei, Taiwan

${ }^{9}$ Institute of Clinical Medicine, School of Medicine, National Yang-Ming University, Taipei, Taiwan

* Vincent Yi-Fong Su and Yung-Tai Chen contributed equally to this manuscript.

\section{CORRESPONDING AUTHOR}

Kun-Ta Chou, MD

Department of Chest Medicine

Taipei Veterans General Hospital

No. 201, Sec. 2, Shi-Pai Rd

Taipei 112, Taiwan

ale1371@yahoo.com.tw
METHODS Using a nationwide database, the Taiwan National Health Insurance Research Database, patients with sleep apnea and age-, sex-, income-, and urbanization-matched control patients who did not have sleep apnea were enrolled between 2000 and 2010. Patients with a prior diagnosis of panic disorder before enrollment were excluded. The 2 cohorts were observed until December 31, 2010. The primary endpoint was occurrence of newly diagnosed panic disorder.

RESULTS A total of 8,704 sleep apnea patients and 34,792 control patients were enrolled. Of the 43,496 patients, 263 (0.60\%) suffered from panic disorder during a mean follow-up period of 3.92 years, including 117 (1.34\%) from the sleep apnea cohort and $146(0.42 \%)$ from the control group. The Kaplan-Meier analysis revealed a predisposition of patients with sleep apnea to develop panic disorder (log-rank test, $P<.001$ ). After multivariate adjustment, the hazard ratio for subsequent panic disorder among the sleep apnea patients was 2.17 (95\% confidence interval, 1.68-2.81; $P<.001)$.

CONCLUSIONS Sleep apnea appears to confer a higher risk for future development of panic disorder.

Ann Fam Med 2015;13:325-330. doi: 10.1370/afm.1815.

\section{INTRODUCTION}

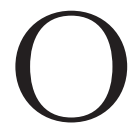
bstructive sleep apnea is a common disorder characterized by repeated episodes of apnea and hypopnea during sleep owing to complete or partial collapse of the upper airway. ${ }^{1}$ Subsequent to apnea/hypopnea, patients with sleep apnea will be awakened by the resulting hypoxemia so that they can resume breathing. Some patients may awake up feeling like they are choking or suffocating, thereby increasing their sense of stress. In the past decade obstructive sleep apnea has been found to be linked to a variety of cardiovascular diseases, neurocognitive dysfunction, and behavioral disorders. ${ }^{2,3}$

Patients with sleep apnea may also have substantial comorbid mental disorders, such as depression, anxiety, bipolar disorder, schizophrenia, post-traumatic stress syndrome, and substance abuse. ${ }^{4}$ Panic disorder is an extreme pole of anxiety disorder with features of recurrent panic attacks. There are, however, few studies addressing the relationship between sleep apnea and panic disorder, and most of these studies are small or lack an appropriate control group. ${ }^{5-9}$ A crossover study enrolling 12 patients with panic disorder who also had obstructive sleep apnea showed that they benefited from continuous positive airway pressure (CPAP) therapy, which decreased the panic attacks and reduced the use of alprazolam, suggesting an interaction of both diseases. ${ }^{10}$ We therefore undertook this nationwide population-based study to elucidate the relationship of sleep apnea and subsequent panic disorder. 


\section{METHODS}

\section{Database}

The National Health Insurance (NHI) is a mandatory universal health insurance program that since 1995 provides comprehensive medical service to almost all Taiwanese citizens. The National Health Research Institute in Miaoli (Taiwan) (http://nhird.nhri.org. tw/en) is in charge of the Taiwan NHI program and maintains the entire insurance claims database, namely, the National Health Insurance Research Database (NHIRD). The NHIRD consists of detailed health care data of more than $99 \%$ of the entire Taiwan's 23 million population. ${ }^{11}$ The data used in this study were retrieved from the Longitudinal Health Insurance Database 2000 (LHID2000), comprised of approximately $1,000,000$ randomly sampled persons who were alive in 2000; all the registration files and medical claims for the reimbursement of these individuals were collected from 1995 to 2010 . The released database has been validated by the National Health Research Institute to be representative of the whole Taiwanese population ${ }^{11}$ and is among the largest populationbased databases in the world; it is a resource for many published studies in Taiwan. ${ }^{12}$ Each patient's personal identifiable information has been encrypted by the National Health Research Institute using a consistent code, allowing linkage of claims belonging to individuals within this database.

\section{Study Sample and Control}

The study was exempt from full review by the Institutional Review Board of Taipei Veterans General Hospital because the data sets consisted of de-identified secondary data. In this study, we enrolled adult patients (aged 20 years and older) who had newly diagnosed sleep apnea (International Classification of Diseases, Ninth Revision, Clinical Modification (ICD-9-CM) codes 780.51, 780.53, 780.57) during 2000 to 2010 as the study cohort (sleep apnea cohort). ${ }^{13}$ The date of enrollment was defined as the date when sleep apnea was initially diagnosed. An age-, sex-, monthly income-, and urbanization-matched group of patients who did not have sleep apnea served as the control group and were randomly selected from the same data sets. In both groups, patients with a medical history of panic disorder (ICD-9-CM codes 300.01, 300.21) before enrollment were excluded.

\section{Variables}

In this research, general data, such as age, sex, monthly income, and urbanization were retrieved and matched between groups. According to past studies, risk factors for panic disorder ${ }^{14}$ and major comorbid conditions ${ }^{3}$ linked to sleep apnea, including hypertension, coronary artery disease, cerebrovas- cular disease, asthma, chronic obstructive pulmonary disease, chronic kidney disease, dyslipidemia, paralysis, irritable bowel syndrome, mitral valve prolapse, premenstrual syndrome, hyperthyroidism, diabetes mellitus, drug abuse, cancer, bipolar disorder, obsessive-compulsive disorder, depression, phobia, and post-traumatic stress disorder, were assessed in our analyses. The Charlson comorbidity index, representative of baseline comorbidity profile, was also calculated and incorporated into the analysis. ${ }^{15}$

\section{Matching}

The control group of patients without sleep apnea was selected at a ratio of 4 control patients per 1 patient with sleep apnea by means of incidence density sampling. ${ }^{16}$ The control group was matched for each individual's age, sex, monthly income, urbanization, and enrollment year. Matching for the age and year of enrollment was allowed within a tolerance range $( \pm 1$ year). For the control group, the start date of follow-up was defined as the first date of clinical visit to a medical facility in the enrollment year.

\section{Main Outcome Measures}

The endpoint of the study was defined as occurrence of panic disorder. All enrollees were observed from the date of enrollment until the first diagnosis of panic disorder or, if they were free of panic disorder, until they died, withdrew from national health insurance, or reached the study end date of December 31, 2010.

\section{Statistical Analysis}

Extraction, matching, and computation of data were performed using the Perl programming language (version 5.12.2). A Microsoft SQL Server 2012 (Microsoft Corp) was used for data linkage, processing, and sampling. Statistical analysis was performed using SPSS 18.0 software (SPSS, Inc). All data were expressed as means plus or minus standard deviations or as a percentage unless otherwise stated. Comparison between 2 groups was made by independent Student's $t$ tests for continuous variables or Pearson's $\chi^{2}$ test for categorical variables, as appropriate. Survival analysis was performed using the Kaplan-Meier method, with significance based on the log-rank test. A Cox proportional hazard model was used for multivariate adjustment. To better elucidate sleep apnea as a dependent risk factor for panic disorder, we performed multivariate adjustments in 5 different models. The hazard ratios were obtained after adjusting age and sex in model 1 and further adjustment for monthly income, urbanization, and outpatient visits in model 2. In models 3 through 5 , additional adjustments were made for psychiatric comorbidities, all comorbidities (as listed in Table 1), 
and the Charlson comorbidity score. Statistical significance was inferred at a 2 -sided $P$ value of $<.05$.

\section{RESULTS}

Figure 1 displays a flowchart of enrollment and follow-up. The basic characteristics of the sleep apnea cohort and the control group are shown in Table 1. Compared with the control group, the sleep apnea cohort had higher percentages of comorbidities, higher Charlson comorbidity scores, and more medical visits in the past year. During a mean follow-up period of 3.92 years, there were more panic disorder events among the sleep apnea cohort as compared with the control group (117 [1.34\%] vs 146 $\left.[0.42 \%]_{i} P<.001\right]$. Sleep apnea patients had a significantly higher risk for incident panic disorder than the comparison group (log-rank test, $P<.001$, Figure 2). Incidences in the sleep apnea cohort and the control group were 33.86 and 10.73 persons per 10,000 person-years, respectively $(P<.001)$. Comparing groups with and without panic disorder, those patients with incident panic disorder were predominately female (54.37\%) and had a higher percentage of hypertension, coronary artery disease, cerebrovascular disease, asthma, chronic obstructive pulmonary disease, chronic kidney disease, dyslipidemia, irritable bowel syndrome, mitral valve prolapse, hyperthyroidism, diabetes mellitus, bipolar disorder, obsessive-compulsive disorder, depression, and phobia.

Multivariate analysis in 5 different models consistently indicated that sleep apnea was independently associated with incident panic disorder (Table 2). The fully adjusted hazard ratio in the model 5 was 2.17 (95\% CI, 1.68-2.81; $P<.001)$. The subgroup analyses are presented in Figures 3 and 4 of the Supplemental Appendix (http://www.annfammed.org/content/13/4/325/supp1/DC1). It is noteworthy that the hazard ratios of all sleep apnea models were statistically significantly higher in their counterparts devoid of a given comorbidity, suggesting an independent role of sleep apnea in development of panic disorder.

\section{DISCUSSION}

In this study, we are able to show that sleep apnea is an independent risk factor for incident panic disorder. Considering the linkage of sleep apnea to important health consequences, our findings may further broaden

Table 1. Demographic and Clinical Characteristics of Patients

\begin{tabular}{|c|c|c|c|}
\hline Characteristics & $\begin{array}{c}\text { Sleep Apnea } \\
(n=8,704)\end{array}$ & $\begin{array}{c}\text { Controls } \\
(n=34,792)\end{array}$ & $\begin{array}{c}P \\
\text { Value }\end{array}$ \\
\hline Mean age, y (SD) & $47.9(15.1)$ & $47.9(15.1)$ & .963 \\
\hline Male, No. (\%) & $5,490(63.07)$ & $21,943(63.07)$ & .993 \\
\hline Monthly income, No. (\%) & & & $>.999$ \\
\hline Dependent & $1,539(17.68)$ & $6,156(17.69)$ & \\
\hline NT\$0-NT\$19,100 & $2,001(22.99)$ & $8,002(23.00)$ & \\
\hline NT\$19,100-NT\$42,000 & $3,713(42.66)$ & $14,852(42.69)$ & \\
\hline$>\mathrm{NT} \$ 42,000^{\mathrm{a}}$ & $1,451(16.67)$ & $5,782(16.62)$ & \\
\hline Urbanization, No. (\%) & & & 1.000 \\
\hline Level 1 (highest) & $5,188(59.60)$ & $20,750(59.64)$ & \\
\hline Level 2 & $2,901(33.33)$ & $11,598(33.33)$ & \\
\hline Level 3 & $503(5.78)$ & $2,003(5.76)$ & \\
\hline Level 4 (lowest) & $112(1.29)$ & $441(1.27)$ & \\
\hline Charlson comorbidity score, No. (\%) & & & $<.001$ \\
\hline 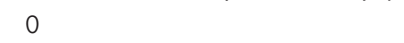 & $1,982(22.77)$ & $14,216(40.86)$ & \\
\hline 1 & $2,074(23.83)$ & $8,323(23.92)$ & \\
\hline 2 & $1,561(17.93)$ & $4,919(14.14)$ & \\
\hline 3 & $1,108(12.73)$ & $2,822(8.11)$ & \\
\hline$\geq 4$ & $1,979(22.74)$ & $4,512(12.97)$ & \\
\hline $\begin{array}{l}\text { Outpatient visits in the past } 1 \text { year, } \\
\text { No. }(\%)\end{array}$ & & & $<.001$ \\
\hline $0-5$ & $122(1.40)$ & $2,854(8.21)$ & \\
\hline $6-10$ & $1,110(12.75)$ & $8,608(24.74)$ & \\
\hline $11-15$ & $1,342(15.42)$ & $6,946(19.96)$ & \\
\hline$>15$ & $6,130(70.43)$ & $16,384(47.09)$ & \\
\hline \multicolumn{4}{|l|}{ Comorbid disease, No. (\%) } \\
\hline Hypertension & $3,429(39.40)$ & $9,204(26.45)$ & $<.001$ \\
\hline Coronary artery disease & $2,198(25.25)$ & $4,992(14.35)$ & $<.001$ \\
\hline Cerebrovascular disease & $1,254(14.41)$ & $2,964(8.52)$ & $<.001$ \\
\hline Asthma & $1,604(18.43)$ & $3,721(10.69)$ & $<.001$ \\
\hline COPD & $2,392(27.48)$ & $5,695(16.37)$ & $<.001$ \\
\hline Chronic kidney disease & $902(10.36)$ & $2,227(6.40)$ & $<.001$ \\
\hline Dyslipidemia & $2,923(33.58)$ & $7,271(20.90)$ & $<.001$ \\
\hline Paralysis & $130(1.49)$ & $353(1.01)$ & $<.001$ \\
\hline Irritable bowel syndrome & $1,476(16.96)$ & $3,405(9.79)$ & $<.001$ \\
\hline Mitral valve prolapse & $505(5.80)$ & $1,072(3.08)$ & $<.001$ \\
\hline Premenstrual syndrome & $57(0.65)$ & $132(0.38)$ & $<.001$ \\
\hline Hyperthyroidism & $464(5.33)$ & $1,081(3.11)$ & $<.001$ \\
\hline Diabetes mellitus & $1,831(21.04)$ & $5,290(15.20)$ & $<.001$ \\
\hline Drug abuse & $332(3.81)$ & $742(2.13)$ & $<.001$ \\
\hline Cancer & $819(9.41)$ & $2,064(5.93)$ & $<.001$ \\
\hline Bipolar disorder & $31(0.36)$ & $60(0.17)$ & .001 \\
\hline Obsessive-compulsive disorder & $44(0.51)$ & $570.16)$ & $<.001$ \\
\hline Depression & $384(4.41)$ & $590(1.70)$ & $<.001$ \\
\hline Phobia & $23(0.26)$ & $37(0.11)$ & $<.001$ \\
\hline PTSD & $9(0.10)$ & $7(0.02)$ & $<.001$ \\
\hline
\end{tabular}


the spectrum of its comorbidities. To our knowledge, this cohort study is the largest probing into this issue. In contrast with other published literature, ${ }^{5-9}$ this study addressed the temporal relationship between sleep

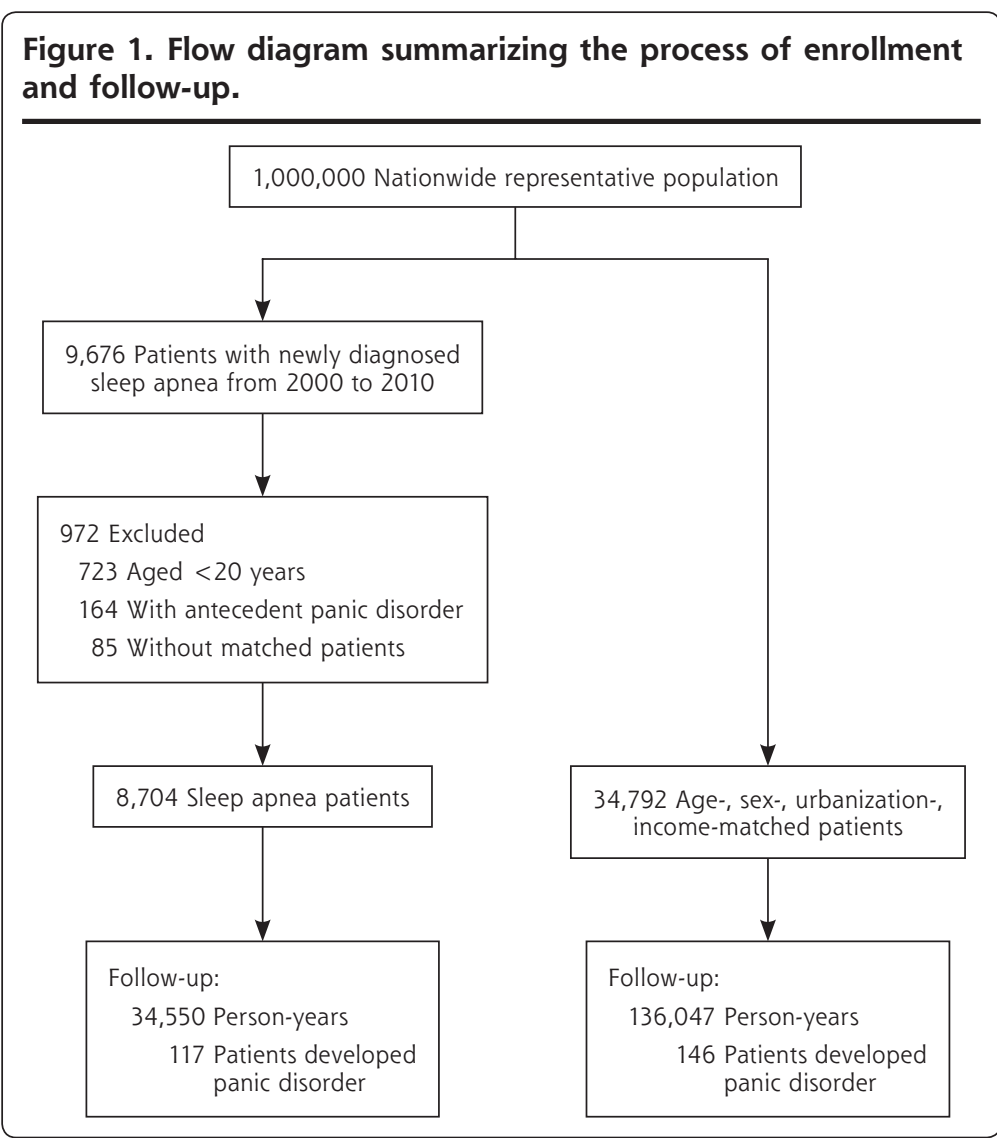

Figure 2. Cumulative incidence of panic disorder in patients with sleep apnea and the matched cohort.

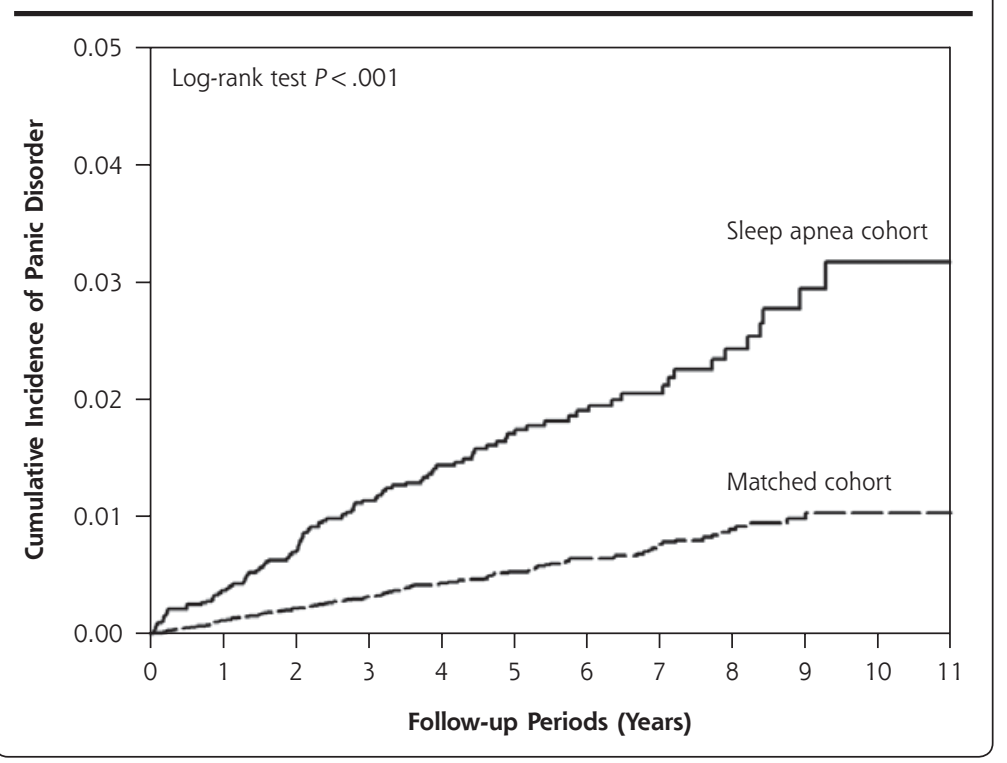

apnea and panic disorder and provided an appropriate control group for comparison as well.

Sleep apnea has been reported to be associated with several mental disorders, including depression, anxiety, posttraumatic stress disorder, psychosis, and bipolar disorders. ${ }^{4,17}$ Interestingly, psychological symptoms may correlate with the degree of hypoxia in such patients and can be improved after CPAP therapy. ${ }^{18,19}$ Patients with coexisting sleep apnea and mental disorders responded less well to antipsychotic medications than did those who did not have sleep apnea. ${ }^{20}$ Thus, identifying patients with overlapping diagnoses of mental disorders and sleep apnea would help to improve their treatment outcomes through more aggressive pharmacotherapy and CPAP therapy.

There have been few studies addressing the relationship of sleep apnea and panic disorder, most were case reports, ${ }^{5.7}$ one was a cross-sectional study $^{8}$ and one was a small-scale casecontrol study, ${ }^{9}$ which has not allowed for a solid conclusion. Our results, derived from a large-scale database, provide a closer look at the relationship of the 2 disorders. Based on our results, sleep apnea conferred a higher risk for panic disorder, even after a relatively short follow-up period (3.9 years) in an older population (age 47.9 years on average). In a randomized crossover study, ${ }_{10}^{10}$ Takaesu et al has shown that CPAP therapy could significantly reduce the frequency of panic attacks, the panic disorder severity scale score, and the use of alprazolam for panic attack in 12 patients with obstructive sleep apnea and panic disorders.

Given that panic disorder in this population may be attenuated after CPAP treatment, the actual prevalence of panic may be higher than found in our current study, a possibility that may further strengthens our conclusion. Moreover, evidence so far favors the inference that the association between sleep apnea and panic disorder results primarily from an influence in one direction (ie, from a history of sleep apnea to first panic attack). The pos- 


\section{Table 2. Serial Multivariate Adjustment Showing Sleep Apnea as a Risk Factor for Incident Panic Disorder}

\begin{tabular}{|c|c|c|c|}
\hline $\begin{array}{l}\text { Adjustment } \\
\text { Model }\end{array}$ & $\begin{array}{c}\text { Hazard } \\
\text { Ratio }\end{array}$ & $95 \% \mathrm{Cl}$ & $\begin{array}{c}P \\
\text { Value }\end{array}$ \\
\hline Crude, unadjusted & 3.16 & $2.47-4.02$ & $<.001$ \\
\hline Model $1^{\mathrm{a}}$ & 3.17 & $2.48-4.04$ & $<.001$ \\
\hline Model $2^{b}$ & 2.62 & $2.04-3.37$ & $<.001$ \\
\hline Model $3^{c}$ & 2.47 & $1.92-3.17$ & $<.001$ \\
\hline Model $4^{d}$ & 2.21 & $1.71-2.86$ & $<.001$ \\
\hline Model $5^{e}$ & 2.17 & $1.68-2.81$ & $<.001$ \\
\hline \multicolumn{4}{|c|}{$\begin{array}{l}\text { adjusted for age, sex. } \\
\text { b Adjusted for age, sex, monthly income, urbanization, outpatient visits. } \\
\text { 'Adjusted for variables in Model } 2 \text { plus psychiatric comorbidities. } \\
\text { d Adjusted for variables in Model } 2 \text { plus all comorbidities in Table } 1 . \\
\text { e Adjusted for variables in Model } 4 \text { plus Charlson comorbidity score. }\end{array}$} \\
\hline
\end{tabular}

sibility of a bidirectional relationship between sleep apnea and panic attacks awaits further exploration.

The link between sleep apnea and panic disorder could be explained in several ways. Frequent arousals from sleep (sleep fragmentation), awakening with feelings of choking or suffocating, and daytime sleepiness have been proposed as mechanisms of anxiety in obstructive sleep apnea. ${ }^{21}$ Additionally, episodes of apnea/hypopnea resulting in intermittent hypoxemia (and possibly hypercapnia) may facilitate oxidative stress-related functional deterioration and central nervous system injury, thereby increasing the risk of panic disorder. ${ }^{22,23}$ Functional and structural neuroimaging studies show that sleep apnea alters brain structure with time, leading to a decrease in gray matter in the hippocampus, frontal lobe, and the anterior cingulate cortex. ${ }^{24-26}$ Damage to similar regions are also seen in the patients with panic disorders. ${ }^{27-30}$ Lastly, hypercapnia may play a role based on the evidence that panic attack can be provoked by inhalation of carbon dioxide. ${ }^{31}$

The strengths of our study are its nationwide, population-based study design and that all respiratory and psychiatric practices were covered in the database which allowed us to trace all cases of newly diagnosed sleep apnea and panic disorder. Additionally, the large sample size in our study offered substantial statistical power for detecting real, even subtle, differences between the 2 cohorts. Because participation in the $\mathrm{NHI}$ is mandatory, and all Taiwanese can use medical care with low copayment, the follow-up of each patient was completed with the lowest referral bias.

There are several limitations in our study. First, diagnoses of sleep apnea and panic disorder that rely on administrative claims data recorded by physicians or hospitals may be less accurate than diagnoses made in a clinical, prospective setting. Sleep apnea is often underdiagnosed, possibly generating a misclassification bias. Even so, the nondifferential misclassification bias was toward the null if the misclassification were corrected, then our positive conclusion would be strengthened, that is, our results would be more significant. Alternatively, some patients with panic disorder might not have used health care, either before or after enrollment, and therefore might not have appeared in the claims data sets as having panic disorder, which may provide a source of bias. Second, patients' body mass index and smoking history, which may affect the propensity to obstructive sleep apnea, were not available in the database. Third, we could not further classify sleep apnea into obstructive type or central type, because the 2001 version of the ICD-9-CM coding did not separate the 2 subtypes of sleep apnea. Even so, obstructive sleep apnea was reported to be the predominant (>90\%) type of sleep apnea by Bixler et al, ${ }_{1}^{32}$ which is compatible with the our results (>99\%) in a previous study. ${ }^{13}$ Finally, the external validity of our findings may be a concern because our patients were almost all Taiwanese. Generalization of our results to non-Asian populations needs further verification.

Sleep apnea may be a risk factor for panic disorder. Clinicians should be aware of panic disorder as a comorbid condition in sleep apnea patients. Future prospective research is needed to confirm our finding and elucidate the possible underlying mechanisms.

To read or post commentaries in response to this article, see it online at http://www.annfammed.org/content/13/4/325.

Key words: sleep apnea syndromes; sleep-disordered breathing; panic disorder

Submitted November 19, 2014; submitted, revised, 22, 2015; accepted May 22, 2015

Supplementary materials: Available at http://www.AnnFamMed. org/content/13/4/325/suppl/DC1/

\section{References}

1. Isono S, Remmers JE, Tanaka A, Sho Y, Sato J, Nishino T. Anatomy of pharynx in patients with obstructive sleep apnea and in normal subjects. J Appl Physiol (1985). 1997;82(4):1319-1326.

2. Bucks RS, Olaithe M, Eastwood P. Neurocognitive function in obstructive sleep apnoea: a meta-review. Respirology. 2013;18(1): 61-70

3. Wang X, Ouyang Y, Wang Z, Zhao G, Liu L, Bi Y. Obstructive sleep apnea and risk of cardiovascular disease and all-cause mortality: a meta-analysis of prospective cohort studies. Int J Cardiol. 2013;169 (3):207-214

4. Lin WC, Winkelman JW. Obstructive sleep apnea and severe mental illness: evolution and consequences. Curr Psychiatry Rep. 2012; 14(5):503-510.

5. Pevernagie D, Mariman A, Vandenbussche $N$, et al. Behavioural hyperventilation as a novel clinical condition associated with central sleep apnoea: a report of three cases. Sleep Med. 2012;13(10): 1317-1320. 
6. Trajanovic NN, Rasool MS, Voloh I, Shapiro CM. Sleep-disordered breathing, cardiac arrhythmia, and panic disorder. J Clin Sleep Med. 2005;1(3):288-289.

7. Enns MW, Stein M, Kryger M. Successful treatment of comorbid panic disorder and sleep apnea with continuous positive airway pressure. Psychosomatics. 1995;36(6):585-586.

8. Edlund MJ, McNamara ME, Millman RP. Sleep apnea and panic attacks. Compr Psychiatry. 1991;32(2):130-132.

9. Stein MB, Millar TW, Larsen DK, Kryger MH. Irregular breathing during sleep in patients with panic disorder. Am J Psychiatry. 1995; 152(8):1168-1173.

10. Takaesu Y, Inoue Y, Komada Y, Kagimura T, limori M. Effects of nasal continuous positive airway pressure on panic disorder comorbid with obstructive sleep apnea syndrome. Sleep Med. 2012;13(2):156-160.

11. Introduction to the National Health Insurance Research Database (NHIRD). Taiwan http://nhird.nhri.org.tw/date_01.html. 2014

12. Publications searched from PubMed using key word "National health insurance database Taiwan. http://nhird.nhri.org.tw/en/ Research.html. 2010

13. Su VY, Liu CJ, Wang HK, et al. Sleep apnea and risk of pneumonia: a nationwide population-based study. CMAJ. 2014;186(6):415-421.

14. Katon WJ. Clinical practice. Panic disorder. N Engl J Med. 2006; 354(22):2360-2367.

15. Charlson ME, Pompei P, Ales KL, MacKenzie CR. A new method of classifying prognostic comorbidity in longitudinal studies: development and validation. J Chronic Dis. 1987;40(5):373-383.

16. Beaumont JJ, Steenland K, Minton A, Meyer S. A computer program for incidence density sampling of controls in case-control studies nested within occupational cohort studies. Am J Epidemiol. 1989;129(1):212-219.

17. Sharafkhaneh A, Giray N, Richardson P, Young T, Hirshkowitz M. Association of psychiatric disorders and sleep apnea in a large cohort. Sleep. 2005;28(11):1405-1411.

18. Bardwell WA, Berry CC, Ancoli-Israel S, Dimsdale JE. Psychological correlates of sleep apnea. J Psychosom Res. 1999;47(6):583-596.

19. Kawahara S, Akashiba T, Akahoshi T, Horie T. Nasal CPAP improves the quality of life and lessens the depressive symptoms in patients with obstructive sleep apnea syndrome. Intern Med. 2005;44(5): 422-427.
20. Gupta MA, Simpson FC. Obstructive sleep apnea and psychiatric disorders: a systematic review. J Clin Sleep Med. 2015;11(2):165-175.

21. Kamphuis J, Meerlo P, Koolhaas JM, Lancel M. Poor sleep as a potential causal factor in aggression and violence. Sleep Med. 2012; 13(4):327-334.

22. Lavie L. Obstructive sleep apnoea syndrome-an oxidative stress disorder. Sleep Med Rev. 2003;7(1):35-51.

23. Riddle DR, ed. Brain Aging: Models, Methods, and Mechanisms. Boca Raton (FL). 2007: CRC Press

24. Zimmerman ME, Aloia MS. A review of neuroimaging in obstructive sleep apnea. J Clin Sleep Med. 2006;2(4):461-471.

25. Gale SD, Hopkins RO. Effects of hypoxia on the brain: neuroimaging and neuropsychological findings following carbon monoxide poisoning and obstructive sleep apnea. J Int Neuropsychol Soc. 2004;10(1):60-71

26. Macey PM, Henderson LA, Macey KE, et al. Brain morphology associated with obstructive sleep apnea. Am J Respir Crit Care Med. 2002;166(10):1382-1387.

27. Asami T, Hayano F, Nakamura $M$, et al. Anterior cingulate cortex volume reduction in patients with panic disorder. Psychiatry Clin Neurosci. 2008;62(3):322-330.

28. Massana G, Serra-Grabulosa JM, Salgado-Pineda P, et al. Parahippocampal gray matter density in panic disorder: a voxel-based morphometric study. Am J Psychiatry. 2003;160(3):566-568.

29. Lai CH. Gray matter deficits in panic disorder: a pilot study of meta-analysis. J Clin Psychopharmacol. 2011;31(3):287-293.

30. Sobanski T, Wagner G, Peikert G, et al. Temporal and right frontal lobe alterations in panic disorder: a quantitative volumetric and voxel-based morphometric MRI study. Psychol Med. 2010;40(11): 1879-1886

31. Amaral JM, Spadaro PT, Pereira VM, Silva AC, Nardi AE. The carbon dioxide challenge test in panic disorder: a systematic review of preclinical and clinical research. Rev Bras Psiquiatr. 2013;35(3):318-331.

32. Bixler EO, Vgontzas AN, Ten Have T, Tyson K, Kales A. Effects of age on sleep apnea in men: I. Prevalence and severity. Am J Respir Crit Care Med. 1998;157(1):144-148. 